\title{
Öklid Uzayında Bir Üçgenin Kenarortaylarının Kesim Noktasının Küresel İmajları ve Çizgiler Uzayında Karşıtları
}

\author{
M. Zihni TEMEL ${ }^{1 *}$ \\ ${ }^{1}$ Dicle Üniversitesi, Mimarlık Fakültesi, Diyarbakır
}

\section{Özet}

Bu çalışmada, Öklid uzayında bir üçgenin kenarortaylarının kesim noktasının küresel imajları vektörel hesapla ispat edilerek çizgiler uzayında karşıtları olan sonuçlar ortaya konmuş ve bu sonuçlar bir uzay altıgeninde verilmiştir.

Anahtar kelimeler: Öklid uzayı, Dual küre, Çizgiler uzayı.

\section{The Spherical Images and to Line Space Corresponding of Center of Gravity of Triangle in the Euclidean Space}

\begin{abstract}
In this paper, the spherical images of the notions of center of gravity of triangle in the Euclidean space were proved by vector calculus. Then passing to line space corresponding notions of a space hexagon were given.
\end{abstract}

Keywords: Euclidean space, Dual spherical, Line space.

\section{Giriş}

Uzay geometrisi için esas temel elamanlar noktalar veya düzlemlerdir. Ancak, J. Plücker (1801-1868) uzay elamanı olarak ilk defa doğrular ve küreleri kullanmıştır. F. Klein (1866-1868) yıllarında Plücker'in 'Doğru çizgilerin uzay elamanı gözüyle bakılmasına dayanan uzayın yeni geometrisi' adlı eserini yayınladi. Bundan önce çizgiler geometrisi, geometrik optikle ilgili olarak W.R Hamilton (18051860) ve diferansiyel geometri açısından da E. Kummer (1810-1893) tarafından geliştirilmiştir. Çizgiler geometrisinin yüzeyler teorisi ile çok sıkı bir ilişkisi olduğu daha sonraları anlaşılıştır. Bu çalışmada $\mathrm{E}$. Study ve W. Blaschke'nin çalışmalarının ışığı altında, düzlem geometride üçgende kenarortaylar teoreminin dual birim küresi üzerindeki kanıtları verilip, bunların çizgiler uzayında karşıtları araştırılacaktır.

\section{Materyal ve Metot}

Çok boyutlu bir uzayda çok parametreli bir lineer alt uzay ailesinin incelenmesi, genel olarak çeşitli modeller oluşturularak yapılmıştır. Alt uzaylar, bu model içerisinde birer nokta olarak kabul edilerek uzayın yeni yapısı inşa edilmiştir. Çizgiler geometri alanında bunun en iyi örneği E. Study tarafından ortaya atılan modeldir. Burada ana fikir geometrinin temel elemanlarından olan nokta ile doğrunun yer değiştirilmesiyle yani dualiteye dayanmaktadır. 


\section{Birim Dual Küre Yüzeyi Üzerindeki Bir Üçgenin Kenarortayları}

Teorem: Birim dual küre yüzeyi üzerine çizilen bir üçgenin üç kenarortayı bir noktada kesişir.

Kanıt: Kenarlara büyük dual daire yayları, köşelere ise noktalar karşılık gelir.

$\vec{A}, \vec{B}, \vec{C}, \overrightarrow{V_{A}}, \overrightarrow{V_{B}}, \overrightarrow{V_{C}}$ ve $\vec{G}$ noktalarının kürenin merkezine göre yer vektörleri olmak üzere ve sırasıyla $\left[\mathrm{B}, \mathrm{C}, \mathrm{V}_{\mathrm{A}}\right],\left[\mathrm{C}, \mathrm{A}, \mathrm{V}_{\mathrm{B}}\right],\left[\mathrm{A}, \mathrm{B}, \mathrm{V}_{\mathrm{C}}\right]$ nokta ölçülerinin aynı düzlemlerde bulunmaları koşulu

$$
\left.\begin{array}{l}
(\vec{B} \Lambda \vec{C}) \cdot \overrightarrow{V_{A}}=0 \\
(\vec{C} \Lambda \vec{A}) \cdot \overrightarrow{V_{B}}=0 \\
(\vec{A} \Lambda \vec{B}) \cdot \overrightarrow{V_{C}}=0
\end{array}\right\}
$$

dır. Birim kürenin merkezi 0 olmak üzere,

$$
\left.\begin{array}{l}
B \hat{O} V_{A}=C \hat{O} V_{A} \rightarrow \vec{B} \cdot \overrightarrow{V_{A}}=\vec{C} \cdot \overrightarrow{V_{A}} \rightarrow \overrightarrow{V_{A}} \cdot(\vec{B}-\vec{C})=0 \\
C \hat{O} V_{B}=A \hat{O} V_{B} \rightarrow \vec{C} \cdot \overrightarrow{V_{B}}=\vec{A} \cdot \overrightarrow{V_{B}} \rightarrow \overrightarrow{V_{B}} \cdot(\vec{C}-\vec{A})=0 \\
A \hat{O} V_{C}=B \hat{O} V_{C} \rightarrow \vec{A} \cdot \overrightarrow{V_{C}}=\vec{B} \cdot \overrightarrow{V_{C}} \rightarrow \overrightarrow{V_{C}} \cdot(\vec{A}-\vec{B})=0
\end{array}\right\}
$$

bağıntıları vardır. $V_{A}^{1}, V_{B}^{1}, V_{C}^{1}$ dual skalerleri $\overrightarrow{V_{A}^{2}}=1, \overrightarrow{V_{B}^{2}}=1, \overrightarrow{V_{C}^{2}}=1$ olacak şekilde seçilerek ve (1), (2) denklemleri kullanılarak

$$
\left.\begin{array}{l}
\overrightarrow{V_{A}}=V_{A}^{1}[(\vec{B} \Lambda \vec{C}) \Lambda(\vec{B}-\overrightarrow{C)}] \\
\overrightarrow{V_{B}}=V_{B}^{1}[(\vec{C} \Lambda \vec{A}) \Lambda(\vec{C}-\overrightarrow{\mathrm{A}})] \\
\overrightarrow{V_{A}}=V_{C}^{1}[(\vec{A} \Lambda \vec{B}) \Lambda(\vec{A}-\overrightarrow{\mathrm{B}})]
\end{array}\right\}
$$

eşitlikleri bulunur.

$$
\vec{B} \cdot \vec{C}=M_{A}, \vec{A} \cdot \vec{C}=M_{B}, \vec{A} \cdot \vec{B}=M_{C}
$$

alınarak, (3) ve (4) ifadelerinden,

$$
\left.\begin{array}{l}
\overrightarrow{V_{A}}=V_{A}^{1}\left(1-M_{A}\right) \cdot(\vec{B}+\vec{C}) \\
\overrightarrow{V_{B}}=V_{B}^{1}\left(1-M_{B}\right) \cdot(\vec{A}+\vec{C}) \\
\overrightarrow{V_{C}}=V_{C}^{1}\left(1-M_{C}\right) \cdot(\vec{A}+\vec{B})
\end{array}\right\}
$$

bulunmuş olur. Burada, 


$$
\left.\begin{array}{c}
V_{A}^{1}\left(1-M_{A}\right)=V_{A} \\
V_{B}^{1}\left(1-M_{B}\right)=V_{B} \\
V_{C}^{1}\left(1-M_{C}\right)=V_{C}
\end{array}\right\}
$$

alınarak

$$
\left.\begin{array}{l}
\overrightarrow{V_{A}}=V_{A}(\vec{B}+\vec{C}) \\
\overrightarrow{V_{B}}=V_{B}(\vec{A}+\vec{C}) \\
\overrightarrow{V_{C}}=V_{C}(\vec{A}+\vec{B})
\end{array}\right\}
$$

kenarortayların yer vektörleri bulunmuş olur.

$\left[A, V_{A}\right],\left[B, V_{B}\right],\left[C, V_{C}\right]$ nokta çiftlerinden geçen büyük dual dairelerin düzlemlerine kürenin merkezinden çıkılan birim dual vektörler, $\mathrm{N}_{\mathrm{A}}, \mathrm{N}_{\mathrm{B}}, \mathrm{N}_{\mathrm{C}}$ dualskalerleri $\overrightarrow{N_{A}^{2}}=1, \overrightarrow{N_{B}^{2}}=1, \overrightarrow{N_{C}^{2}}=1$ ile belli olmak üzere

$$
\left.\begin{array}{l}
\overrightarrow{N_{A}}=N_{A}\left(\overrightarrow{V_{A}} \Lambda \vec{A}\right) \\
\overrightarrow{N_{B}}=N_{B}\left(\overrightarrow{V_{B}} \Lambda \vec{B}\right) \\
\overrightarrow{N_{C}}=N_{C}\left(\overrightarrow{V_{C}} \Lambda \vec{C}\right)
\end{array}\right\}
$$

şeklindedir. (8)'de (7) eşitliklerini kullanarak ve $N=V_{A} V_{B} V_{C} N_{A} N_{B} N_{C}\left(1-M_{A}\right)\left(1-M_{B}\right)\left(1-M_{C}\right)$ olmak üzere $\left(\overrightarrow{N_{A}}, \overrightarrow{N_{B}}, \overrightarrow{N_{C}}\right)$ karma çarpımı oluşturulursa

$\left(\overrightarrow{N_{A}}, \overrightarrow{N_{B}}, \overrightarrow{N_{C}}\right)=N((\vec{B}+\vec{C}) \Lambda \vec{A},(\vec{A}+\vec{C}) \Lambda \vec{B},(\vec{A}+\vec{B}) \Lambda \vec{C})$ bulunur. Determinant özelliklerinden bu son bağıntı

$$
\left(\overrightarrow{N_{A}}, \overrightarrow{N_{B}}, \overrightarrow{N_{C}}\right)=0
$$

eşitliğini verir. (9) ifadesi, birim dual küre üzerine çizilen bir üçgenin kenar ortalarının bir noktada kesişmesi koşuludur.

Şimdi bu teoremin $I R^{3}$ çizgiler uzayında karşıtını araştıralım. Birim dual küresi üzerindeki her bir noktanın çizgiler uzayında bir doğruya karşılık geleceğini göz önüne alarak,

$\mathrm{B}, \mathrm{C}, \mathrm{V}_{\mathrm{A}}$ nın düzlemine dik olarak çıkılan birim vektör

$\mathrm{C}, \mathrm{A}, \mathrm{V}_{\mathrm{B}}$ nin düzlemine dik olarak çıkılan birim vektör

$\overrightarrow{V_{2}}$ 
$\mathrm{A}, \mathrm{B}, \mathrm{V}_{\mathrm{C}}$ nin düzlemine dik olarak çıkılan birim vektör

$\mathrm{A}, \mathrm{V}_{\mathrm{A}}, \mathrm{G}$ nin düzlemine dik olarak çıkılan birim vektör

$\mathrm{B}, \mathrm{V}_{\mathrm{B}}, \mathrm{G}$ nin düzlemine dik olarak çıkılan birim vektör

$\mathrm{C}, \mathrm{V}_{\mathrm{C}}, \mathrm{G}$ nin düzlemine dik olarak çıkılan birim vektör

olsun. Buna göre aşağıdaki bağıntılar yazılır,

(I) den, $\left.\left.\left.a_{1}\right) \overrightarrow{V_{1}} \perp \vec{B} a_{2}\right) \overrightarrow{V_{1}} \perp \vec{C} a_{3}\right) \overrightarrow{V_{1}} \perp \overrightarrow{V_{A}}$

(II)den, $\left.\left.\left.b_{1}\right) \overrightarrow{V_{2}} \perp \vec{C} b_{2}\right) \overrightarrow{V_{2}} \perp \vec{A} b_{3}\right) \overrightarrow{V_{2}} \perp \overrightarrow{V_{B}}$

(III) ten, $\left.\left.\left.c_{1}\right) \overrightarrow{V_{3}} \perp \vec{A} c_{2}\right) \overrightarrow{V_{3}} \perp \vec{B} c_{3}\right) \overrightarrow{V_{3}} \perp \overrightarrow{V_{C}}$

IV) ten, $\left.\left.\left.d_{1}\right) \overrightarrow{V_{4}} \perp \vec{A} d_{2}\right) \overrightarrow{V_{4}} \perp \overrightarrow{V_{A}} d_{3}\right) \overrightarrow{V_{4}} \perp \vec{G}$

(V) ten, $\left.\left.\left.e_{1}\right) \overrightarrow{V_{5}} \perp \vec{B} e_{2}\right) \overrightarrow{V_{5}} \perp \overrightarrow{V_{B}} e_{3}\right) \overrightarrow{V_{5}} \perp \vec{G}$

(VI)dan, $\left.\left.\left.f_{1}\right) \overrightarrow{V_{6}} \perp \vec{C} f_{2}\right) \overrightarrow{V_{6}} \perp \overrightarrow{V_{C}} f_{3}\right) \overrightarrow{V_{6}} \perp \vec{G}$

Böylece, $a_{1}, a_{2}, b_{1}, b_{2}, c_{1}, c_{2}$ bağıntılarından

(B) ve (C) doğrularının ortak dikmesi $\left(\mathrm{V}_{1}\right)$

(C) ve (A) doğrularının ortak dikmesi $\left(\mathrm{V}_{2}\right)$

(A) ve (B) doğrularının ortak dikmesi $\left(\mathrm{V}_{3}\right)$

bulunur. $\mathrm{A}_{1} \mathrm{~A}_{2} \mathrm{~A}_{3} \mathrm{~A}_{4} \mathrm{~A}_{5} \mathrm{~A}_{6}$ dik uzay altıgeni oluşur. (1) denklemlerinde ve $\mathrm{a}_{3}, \mathrm{~b}_{3}, \mathrm{c}_{3}$ bağıntılarından

$\left(\mathrm{V}_{\mathrm{A}}\right),(\mathrm{B})$ ve $(\mathrm{C})$ ile eşit açı yaparak $\left(\mathrm{V}_{1}\right)$ e orta noktada dik

$\left(\mathrm{V}_{\mathrm{B}}\right),(\mathrm{C})$ ve $(\mathrm{B})$ ile eşit açı yaparak $\left(\mathrm{V}_{2}\right)$ e orta noktada dik

$\left(V_{C}\right),(A)$ ve $(B)$ ile eşit açı yaparak $\left(V_{3}\right)$ e orta noktada dik

elde edilir.(VIII ) den $\left(\mathrm{V}_{\mathrm{A}}\right),\left(\mathrm{V}_{\mathrm{B}}\right),\left(\mathrm{V}_{\mathrm{C}}\right)$ doğruları oluşur.

$\mathrm{d}_{1}, \mathrm{~d}_{2}, \mathrm{e}_{1}, \mathrm{e}_{2}, \mathrm{f}_{1}, \mathrm{f}_{2}$ bağıntılarından

(A) ve $\left(\mathrm{V}_{\mathrm{A}}\right)$ nın ortak dikmesi $\left(\mathrm{V}_{4}\right)$

(B) ve $\left(\mathrm{V}_{\mathrm{B}}\right)$ nın ortak dikmesi $\left(\mathrm{V}_{5}\right)$

(C) ve $\left(\mathrm{V}_{\mathrm{C}}\right)$ nin ortak dikmesi $\left(\mathrm{V}_{6}\right)$

(IX)

bulunur.( IX ) dan $\mathrm{A}_{7} \mathrm{~A}_{8} \mathrm{~A}_{9} \mathrm{~A}_{10} \mathrm{~A}_{11} \mathrm{~A}_{12}$ uzay altıgeninin karş1lıklı kenarlarının ortak dikmeleri elde edilir. $\mathrm{d}_{3}, \mathrm{e}_{3}, \mathrm{f}_{3}$ bağıntılarından da $\left(\mathrm{V}_{4}\right),\left(\mathrm{V}_{5}\right),\left(\mathrm{V}_{6}\right)$ doğrularının ortak dikmesi $(\mathrm{G})$

bulunur. Sırasıyla VII, VIII, IX, X özellikleri kullanılarak aşağıdaki sonuç elde edilir:

Sonuç : IR ${ }^{3}$ çizgiler uzayında bir dik uzay altıgeninin $\left(\mathrm{A}_{1} \mathrm{~A}_{2} \mathrm{~A}_{3} \mathrm{~A}_{4} \mathrm{~A}_{5} \mathrm{~A}_{6}\right)$ aralıklı üç kenarının $\left(\left(\mathrm{V}_{1}\right)\right.$, $\left.\left(\mathrm{V}_{2}\right),\left(\mathrm{V}_{3}\right)\right)$ orta noktalarından $\left(\mathrm{A}_{13}, \mathrm{~A}_{14}, \mathrm{~A}_{15}\right)$ bu kenarlara bitişik noktalarda ((B) ile $(\mathrm{C}),(\mathrm{C})$ ile $(\mathrm{A})$, (A) ile $(B))$ eşit açılar yapacak şekilde çıkılan dikmelerin $\left(\left(V_{A}\right),\left(V_{B}\right),\left(V_{C}\right)\right)$, karşılarındaki kenarlarla $((A),(B),(C))$ ortak dikmelerinin $\left(\left(V_{4}\right),\left(V_{5}\right),\left(V_{6}\right)\right)$, bir ortak dikmesi $(G)$ vardır.

Şimdi ABC küresel üçgeninin ağırlık merkezi $(G)$ yi hesaplayalım.

$\left[\mathrm{G}, \mathrm{A}, \mathrm{V}_{\mathrm{A}}\right]$ ve $\left[\mathrm{G}, \mathrm{B}, \mathrm{V}_{\mathrm{B}}\right]$ nokta üçlülerinin aynı düzlemlerde bulunmaları nedeniyle, 


$$
\left.\begin{array}{l}
\vec{G} \cdot\left(\vec{A} \Lambda \overrightarrow{V_{A}}\right)=0 \\
\vec{G} \cdot\left(\vec{B} \Lambda \overrightarrow{V_{B}}\right)=0
\end{array}\right\}
$$

bağıntıları vardır. $\mathrm{G}_{1}$ dual skaleri $\overrightarrow{G_{2}}=1$ ile belli olmak üzere (10) bağıntılarından

$$
\vec{G}=G_{1}\left[\left(\vec{A} \Lambda \overrightarrow{V_{A}}\right) \Lambda\left(\vec{B} \Lambda \overrightarrow{V_{B}}\right)\right]
$$

bulunur. (7) denklemlerini de göz önüne alarak ve $\vec{G}=G_{1} \overrightarrow{V_{A}} \overrightarrow{V_{B}}\left(1-M_{A}\right)\left(1-M_{B}\right)(\vec{A}, \vec{B}, \vec{C})$ dual skalerini kullanarak (11) ifadesi

$$
\vec{G}=G(\vec{A}+\vec{B}+\vec{C})
$$

şeklinde G nin konumu belirlenmiş olur.

\section{Sonuç ve Öneriler}

Bu çalışmada, farklı iki geometrik yapı içerisindeki kavramlar karşılaştırılarak her iki geometride de geçerli olan teorilerin kendi yapıları içerisindeki ifadelerin, diğer geometrik uzaydaki karşılığı tanımlanarak daha kapsamlı sonuçlara ulaşılmıştır. Bu çalışmada yapılan Öklid uzayındaki bir üçgenin kenarortay teoreminin birim dual küre üzerinde geçerli olduğunu kanıtlayıp ters projeksiyonla çizgiler uzayının daha karmaşık orijinal teoremi elde edilmiştir.

\section{Kaynaklar}

1. Blaschke W. 1950. Einführung in die Differentialgeometrie. Springer Verlag, Berlin, Heidelberg, New York.

2. Guggenheimer H.W. 1963. Differential Geometry. McGraw-Hill, New York.

3. Laugwitz D. 1960. Differentialgeometrie. B.G. Teubner Verlagsgesellschaft, Stuttgart.

4. Gromov M. 1983. Filling Riemannian Manifolds, J. Differential Geom., 18 (1): 1-147.

5. Rochowski M. 1992. Special Problems of Surface Theory in the Euclidean 3-Dimensional Space. Demonstratio Mathematica, Warszawska. 